\title{
The study of High Accuracy Inner Gear Finishing Method
}

\author{
Hisatoshi KOJIMA ${ }^{1}$, Toshiya HAYASHI ${ }^{2}$, Chunye PENG Katsumi NAGASAKA \\ ${ }^{1}$ Production Eng. R\&D Dept., DENSO CORPORATION, Japan, HISATOSHI_KOJIMA@denso.co.jp \\ ${ }^{2}$ Driving Assist \& Safety Mfg. Dept., DENSO CORPORATION, Japan, TOSHIYA_HAYASHI@denso.co.jp
}

\begin{abstract}
:
This paper introduced a new mass production method for inner gear grinding with high precision and low cost. The method uses an outer-gear-shape grindstone to gear with and grind the inner-gear-shape workpiece under a fixed pressure while rotating. To grind the whole inner-gear surface precisely, the tangency mode between the grindstone and workpiece is analyzed by numerical simulation. Furthermore, by searching, analyzing and modifying the relationship between the finished gear profile and the corresponding applied pressure, the final profile can be tuned and thus high accuracy can be achieved.
\end{abstract}

Keywords: High-accuracy, Gear grinding, Gear shape grindstone, Accompanied rotation

\section{Introduction}

In recent years, increasing demands such as higher safety performance, better environment performance (lower waste gas emissions), smaller size and lighter weights are cast to the automobiles. Accordingly, those parts assembled into the automobiles are also asked to be more and more compact. As a result, the automobile parts become more complex because of smaller diameters, deeper holes and smaller thickness. These factors also limited the diameters/sizes and then lowered the rigidities of both the cutting tools and the work-pieces. Even the required tolerances are kept as same as those in the past, it becomes more difficult to maintain the same machining precision with the same or a lower cost. This situation has significantly increased the urgency for developing completely new machining technologies than merely improving those existing ones. To fulfill this requirement, we have developed a wide range of new machining technologies by combining several DENSO original technologies with some other well-known ones. Concerning the requirements to our products, we focused mainly on high accuracy machining technologies that related to seal performance and fuel injection performance such as precise deep hole opening, precise profile making, precise shape making of small-thickness parts, and fine hole opening. Fig. 1 shows some representative products manufactured by our new developed machining technologies. In this paper, we will introduce a new mass-production method for inner gear surface grinding. The method can also be applied to high-precision profile grindings for any other irregular inner shapes. The workpiece to be grinded has a small-diameter $(20 \mathrm{~mm})$ internal-gear surface that demands of gauge-level precision for the whole tooth surfaces including the tip and the root, which makes it extremely hard to be grinded by any conventional method.

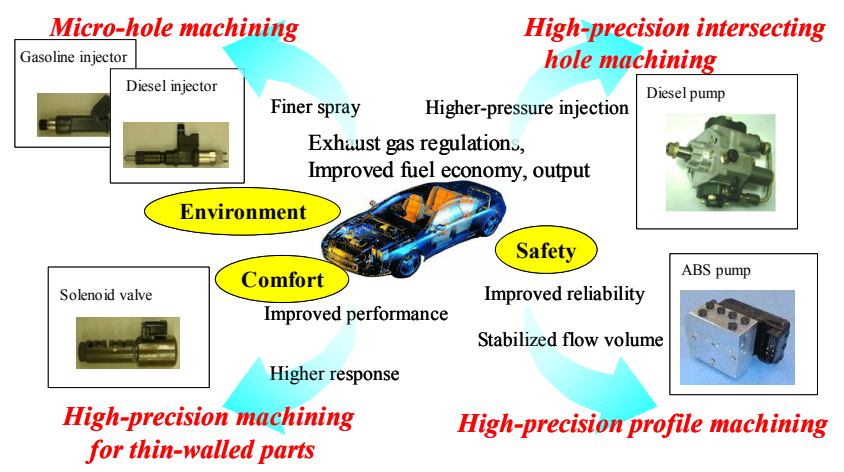

Fig. 1 Manufactured products with high-precisions

\section{New Developed Inner Gear Grinding Method}

\subsection{Problems of conventional method}

The conventional methods for grinding this inner gear surface may use a revolving gear shape grindstone which is smaller than the inside diameter of the workpiece or use a cylindrical grindstone whose radius is smaller than the minimum radius of the workpiece and runs through the whole gear surface ${ }^{[1]}$. However, either method grinds those gear teeth one by one, accordingly the machining precision will differs each other because of 
the positioning and feeding accuracy. Furthermore, a high-precision index positioning and feeding system is needed and the cost of the equipment increases largely that becomes difficult to be applied to mass production.

\subsection{New Machining Method}

Fig. 2 illustrates the principle of a new developed machining method. The gear to be grind (hereinafter called the "workpiece") is supported by a V-shape rest, and a designed gear-shape grindstone is set gearing with it. The machining is carried out by pressing the grindstone onto the workpiece surface when rotating, at the same time the grindstone is oscillating at a quite high speed along its axial direction.

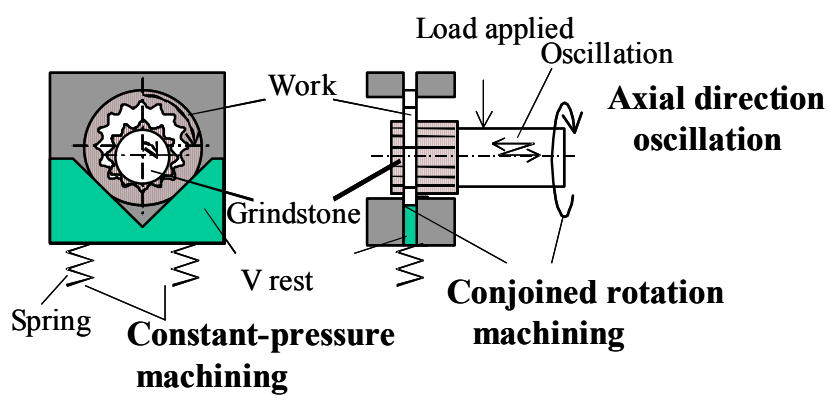

Fig. 2 Principle of the schemed new machining method

One advantage of this new machining method is that by changing the number of the grindstone teeth, each workpiece tooth contacts with different grindstone tooth in every revolution. Thus, during machining each grindstone tooth is forced to contacts with every workpiece tooth, and the machining conditions for every workpiece tooth are all of the same, then the variation in the shape of each workpiece tooth can be reduced.

Another advantage of this method is that the work is supported by a V rest but not using any chuck. Also the workpiece is forced to revolve in conjunction with the rotation of the grindstone. These factors mean that the high-precision positioning mechanism and the chuck mechanism to position the grindstone and the work can be eliminated. Moreover, the feeding mechanism can be eliminated either by adopting a constant-pressure imposing system, therefore the equipment cost can be reduced largely.

\section{To Achieve High Machining Precision}

Studying of this new grinding method, we found that because the grindstone and the workpiece are pressed against each other, the locations that bearing larger forces will be removed more volumes than those smaller ones. Therefore it is important to control the forces equally acting on each location in order to improve the machining precision.

\subsection{Influences of machining forces generated by conjoined rotation}

Fig. 3 shows the forces acting on the workpiece in this machining method. It shows the pressing force and rotational force generated by the grindstone, as well as the resistance of the $\mathrm{V}$ rest to the rotational force, or rotational resistance acting on the workpiece.

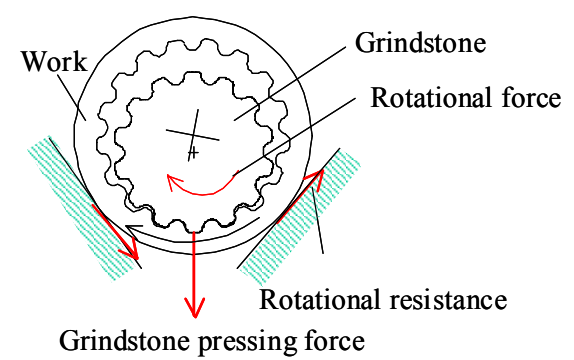

Fig. 3 Forces acting on the workpiece in the new machining method

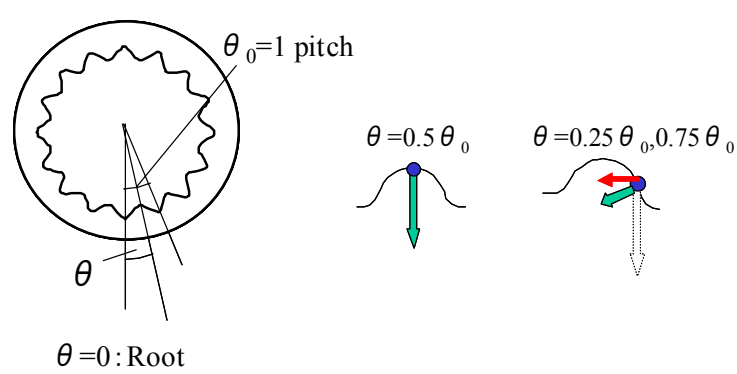

Fig. 4 Variation in machining force at each machined location

Fig. 4 shows how the forces acting on the work change with the location on the tooth surface. Here, $\theta$ is defined as the pitch angle, and the position of $\theta=0$ represents a tooth root. Also, $\theta_{0}$ represents the angle of one pitch. As shown in Fig. 4, when the tooth tip or the tooth root of the workpiece is being machined, that is, when $\theta=0,0.5 \theta_{0}$, or $1 \theta_{0}$, the direction of grindstone pressure matches the normal direction of the tooth surface. The grindstone pressing force therefore acts as the main machining force (hereinafter called the "normal force on the tooth surface") acting on the workpiece. However, when the side of the tooth is machined, the grindstone pressing force is acting on the inclined tooth surface, and thus generates a horizontal force. If the rotational resistance is low at this time, this horizontal force will causes the workpiece to shift away from the grindstone to the rotation direction, thus the normal force on the side tooth surface is lowered and the machining precision will be worsened. Therefore, to improve the machining precision, it is necessary to impose additional rotational resistance to the workpiece. 


\subsection{Method of imposing rotational resistance}

A method of imposing additional rotational resistance was designed adopting a friction brake. The situation of additional rotational resistance imposing on the workpiece is shown in Fig. 5. The normal force acting on the tooth surface can be calculated by Equation (1). Here, $\theta_{1}$ is the angle between the direction of grindstone pressure and the tooth surface tangent line, which can be derived from the pitch angle $\theta$ and the designed shape of the tooth surface. Fig. 6 shows the relationships between each location on the workpiece tooth profile and the corresponding normal force acting, which are calculated by Equation (1) by applying three values of different rotational resistance from low to high. The horizontal axis indicates the tooth pitch, which is made dimensionless by $\theta_{0}$, and the vertical axis indicates the normal force acting on the tooth surface, also made dimensionless by the pressing force $\mathrm{p}$ of the grindstone. From Fig. 6, when the rotational resistance is low, the shifting of the workpiece away from the grindstone causes the normal force on the tooth surface lowered near $\theta=0.25 \theta_{0}$ and $\theta=0.75 \theta_{0}$, which correspond to the sides of the tooth. In contrast, when the rotational resistance becomes high, it can be seen that the normal force on the tooth surface increases largely. Moreover, we can see that the normal force on the tooth surface decreases at those protruding locations near the sides of each tooth. This phenomenon is caused by angle $\theta_{1}$, which is determined by the designed tooth profile. And this problem will be solved by our future study.

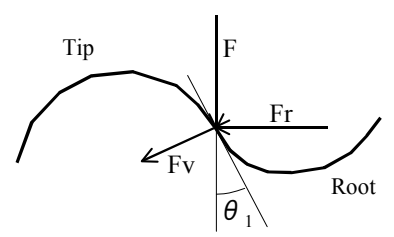

F : Grindstone pressing force

$\mathrm{Fr}$ : Force acting in direction of

rotation due to rotational resistance

$\mathrm{Fv}:$ Normal force on tooth surface
$\theta_{1}:$ Angle between grindstone pressing

direction and tooth surface tangent line

$\mathrm{Fv}=\mathrm{F} \sin \theta_{1}+\mathrm{Fr} \cos \theta_{1}$

Fig. 5 Relationship between Each Machined Location and Forces Acting on It

\section{Verified by Practical Machining}

Fig. 7 shows the machining equipment used in practical machining. The rotational resistance is imposed onto the workpiece by a brake from above, which pressing the work against the $\mathrm{V}$ rest. Note that the brake/V rest system is supported independent from the grindstone axis, so the pressing force of the brake has no effect on the pressing force of the grindstone. Fig. 8 shows the photographs of the workpiece surface before and after machining. It can be seen that after grinding, no black skin remains on any grinded surfaces including the tips, roots, and side faces. This indicated that grinding of the whole tooth surface has been achieved.

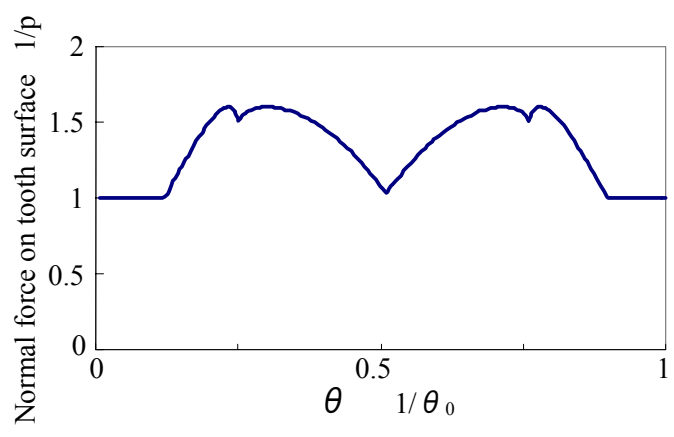

(a) High rotational resistance

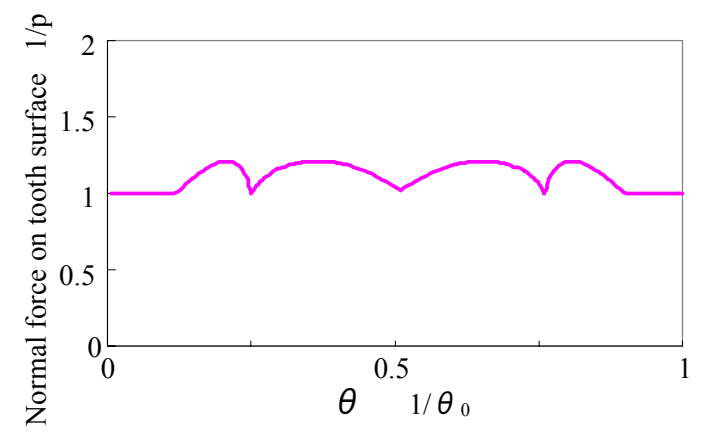

(b) Medium rotational resistance

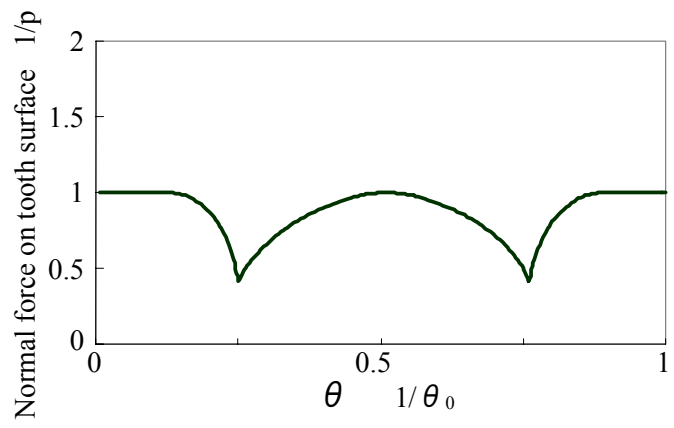

(c) Low rotational resistance

Fig. 6 Relationship between each machined location and normal force on tooth surface

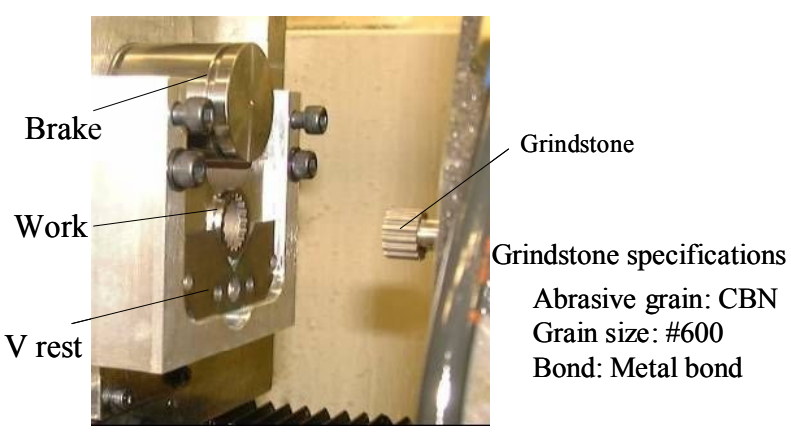

Fig. 7 Machining equipment 
Fig. 9 shows the profile precision of one tooth before and after the machining. The horizontal axis indicates the position on the tooth, as that in Fig. 6, while the vertical axis indicates the deviations from the designed values. A larger deviation shows that more volumes were removed. Measurements were taken at 200 points per tooth with an optical measurement instrument. It can be seen that even the profiles remains almost the same before machining, when after grinding, the deviations varies largely according to the magnitude of applied rotational resistance. We can also found that after machining some grinding remains appeared at those protruding locations where the normal force on the tooth surface decreases. It is clear that these grinding remains fits well with the distribution state of controlled normal force shown in Fig.6. The grinding remains are also considered being determined by the designed tooth profile. The above results indicate that the controlled distribution of the normal force on the tooth surface and the profile of the tooth after machining match perfectly.

\section{Conclusion}

A machining method that uses new mechanisms of conjoined rotation between the grindstone and the workpiece and involves constant-pressure pressing has been developed. This method is applied as a mass grinding production method with high precision and low cost for grinding the whole surface of a small-diameter inner gear. It has also been verified that this new method makes it possible to grind the tooth surface more precisely and can achieve desired machined gear profile by using a brake to impose additional rotational resistance.

\section{Reference}

[1] Processing technology data file, TECHNICAL RESEARCH INSTITUTE Japan Society for the Promotion of Machine Industry, 1996, 22-0114-42-01, 20-0107-52-01

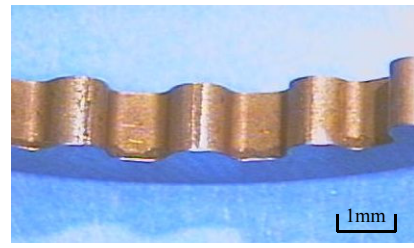

Before machining

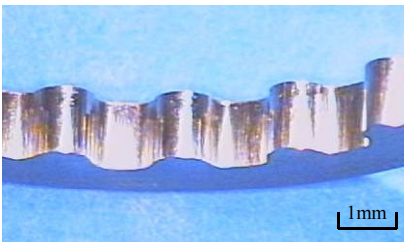

After machining
Fig. 8 Work surface before and after machining

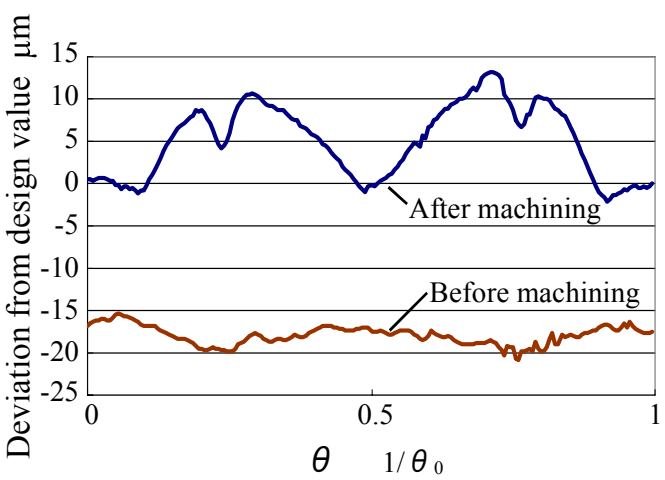

(a) High rotational resistance

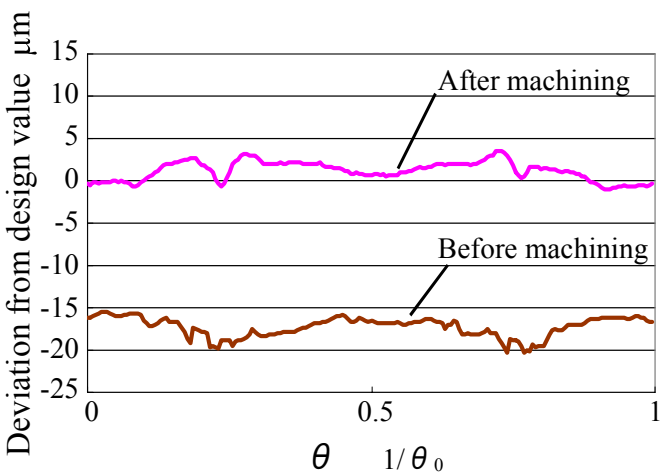

(b) Medium rotational resistance

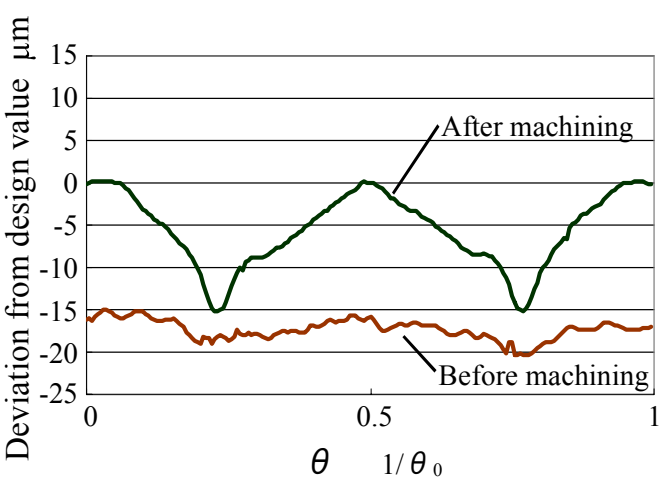

(c) Low rotational resistance

Fig. 9 Shape precision of work before and after machining 